\title{
Permanent Magnet Variable Flux Sources
}

\author{
O.Cugat, P.Hansson and J.M.D.Coey \\ Physics Department, Trinity College, Dublin 2, Ireland.
}

\begin{abstract}
Rotatable transversely-magnetized permanent magnet rods can be used to generate variable, uniform magnetic fields or field gradients. A four-rod device for use at the specimen stage of an optical microscope is described where the field can be in-plane or perpendicular to the stage. The maximum flux density ranges from $36 \mathrm{mT}(360 \mathrm{G})$ for isotropic ferrite to $210 \mathrm{mT}(2.1 \mathrm{kG})$ for sintered $\mathrm{Nd}$ Fe-B. Calculated and measured field profiles are in good agreement. Homogeneity is better than $1 \%$ of maximum in a region of diameter $7 \mathrm{~mm}$ or $3.5 \mathrm{~mm}$ at maximum and zero field, respectively.
\end{abstract}

A variable magnetic field is a useful specimen environment for many types of instrumentation. In microscopy there are applications such as magnetic domain observation, examination of magnetotactic micro-organisms or study of the behaviour of ferrofluids. A variable magnetic field gradient can be used to apply force selectively to magnetically-labelled species and thereby displace them at will in the field of view.

Variable magnetic fields are traditionally generated by current-carrying coils. A pair of Helmholtz coils whose radius $a$ is equal to their separation generates a uniform field of magnitude $\mathrm{H}=8 /(5 \sqrt{5}) \mathrm{nI} / \mathrm{a}$ where $\mathrm{I}$ is the current and $\mathrm{n}$ the number of turns on each coil. For example, if $a=5 \mathrm{~cm}, I=10$ amps and $\mathrm{n}=100$, then $\mathrm{H}=14 \mathrm{kA} / \mathrm{m}$. The corresponding flux density $B_{0}=\mu_{0} \mathrm{H}$ is $18 \mathrm{mT}$. Helmholtz coils are useful for generating weak fields and field gradients, especially along the optical axis. Field intensities of order $100 \mathrm{mT}$ demand bulky coils with many turns carrying large currents, which in turn require substantial power supplies and water cooling.

A more versatile and convenient solution to the problem of creating a variable magnetic field in a region of space is to use rotatable permanent magnets. The field in $\mathrm{A} / \mathrm{m}$ due to a point dipole $\mu \mathrm{J} / \mathrm{T}$ at any point $(r, \theta)$ is

$$
H_{p}=\left(\mu / 4 \pi r^{3}\right)\{2 \cos \theta \hat{r}+\sin \theta \hat{\theta}\}
$$

where $\hat{\mathbf{r}}$ and $\hat{\theta}$ are unit vectors in the radial and transverse directions, whereas the field due to an extended line dipole $\lambda$ $\mathrm{J} / \mathrm{Tm}$ at any point in the plane perpendicular to its axis is

$$
H_{1}=\left(\lambda / 2 \pi r^{2}\right)\{\cos \theta \hat{\mathbf{r}}+\sin \theta \hat{\theta}\}
$$

The variation in field for the two cases is compared in Figure 1. In the second case the magnitude of the field is independent of $\theta$, and lies in a direction making an angle $2 \theta$ with the dipole. The independence of $\mathrm{IHI}$ on $\theta$ can be exploited to design extended permanent magnet flux sources which deliver uniform magnetic fields with virtually unobstructed access [1]. A key feature of modern permanent magnets is that the fields of different magnets can be superposed without modifying the characteristics of each flux source [2]. Equation 2 is the basis of the design of the 'dipole ring' or 'magic cylinder' which generates a uniform transverse field when segments at an

Manuscript received 31st March 1994

J.M.D.Coey, e-mail jcoey@vax1.tcd.ie; fax 353-1-6711759.

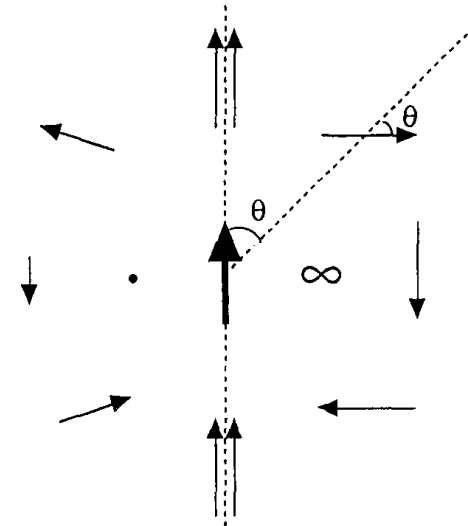

Fig 1: Illustration of the magnitude and direction of the magnetic field produced at a distance $\mathrm{r}$ from a point dipole (left) and a long line dipole perpendicular to the plane of the paper (right). The dipole in each case is oriented vertically, as shown

angular position $\alpha$ in the ring are magnetized in directions making an angle $2 \alpha$ with the field. Leupold and Potenziani [3] suggested the use of two similar concentric cylinders which rotate about their common axis to generate variable uniform fields, and we have recently implemented this concept in a 1.2 T compact vibrating-sample magnetometer [4].

Here we propose a novel class of variable flux sources which are based on rotatable magnetic rods magnetized across a diameter. If we consider a single long rod of radius $1 \mathrm{~cm}$ made of a permanent magnet material of polarization $\mathrm{J}_{\mathrm{r}}=1 \mathrm{~T}$, the line dipole moment is $\lambda=250 \mathrm{~J} / \mathrm{T} / \mathrm{m}$. The field at a distance of $3 \mathrm{~cm}$ from the magnet has magnitude $44 \mathrm{kA} / \mathrm{m}$, corresponding to a flux density of $56 \mathrm{mT}$. The value at the surface of the cylinder is $500 \mathrm{mT}$. If several rods are arranged with their axes on a cylinder, their contributions to the magnetic field in the enclosed space add vectorially, and the magnitude and direction of the field can be varied by rotating the cylinders in unison by means of appropriate gearing.

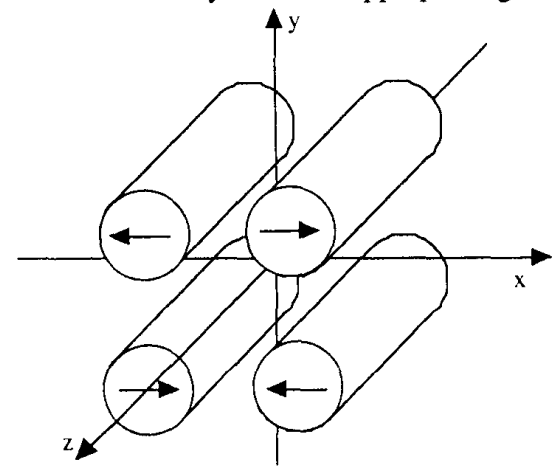

Fig 2: Layout of a variable flux source composed of four rotating cylindrical magnets - the "magic mangle". 


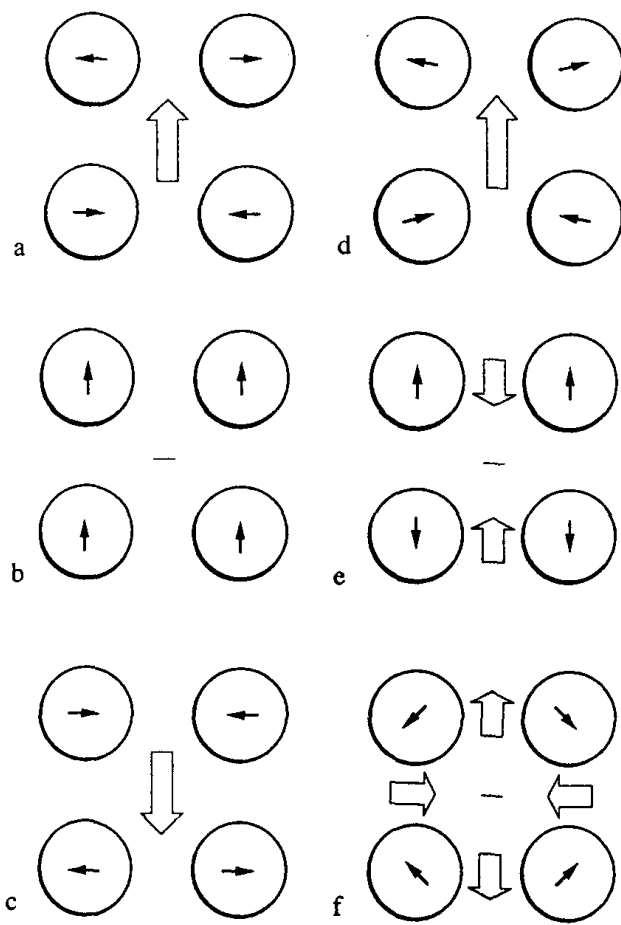

Fig 3: Orientations of four long magnetic rods which produce (a) maximum field $B_{\max }$, (b) zero field and (c) minimum field $-B_{\max }$. The adjustment needed to optimize the maximum field with short rods is shown in (d). Configuration (e) produces a magnetic field gradient at the centre, and ( $f$ ) gives a quadrupole field.

\section{TABLE 1}

Typical remanent polarization for commercial magnets, and the flux density expected for an ideal mangle with $(\mathrm{a} / \mathrm{d})=0.25$

Type

$$
J_{r}(T)
$$

$B_{\max }(m T)$

Isotropic ferrite

Oriented ferrite

0.2
0.4

0.4
0.6

1.2

Sintered Nd-Fe-B

50
100
150
300

Simple designs use four, six or eight rods geared so that alternate rods rotate in opposite directions. Here we discuss a four-rod device, the 'magic mangle', where the centres of the rods lie on a square of side $d$ as shown in Figure 2. The field at the centre is perpendicular to the axis of the device. Maximum flux density at the centre is achieved when the rods are oriented as shown in Figure 3(a) so that the contributions from all four lie in the $y$-direction. Then

$$
\mathrm{B}_{\max }=4(\mathrm{a} / \mathrm{d})^{2} \mathrm{~J}_{\mathrm{r}}
$$

The maximum value of $a / d$ is obviously $1 / 2$, at contact, and then the flux density $B_{\max }=J_{r}$, To achieve the good vertical and horizontal access required for a microscope stage it is necessary to choose $a / d \sim 1 / 4$. The remanent polarization for some typical permanent magnets is summarized in Table 1,

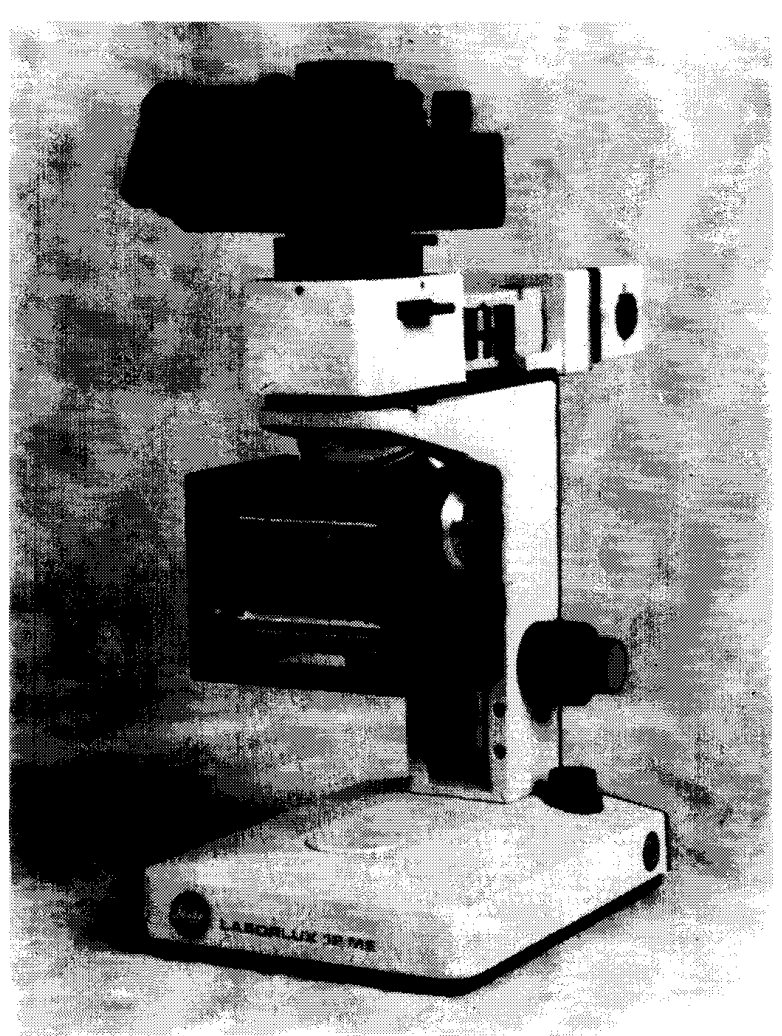

Fig 4: Illustration of the magic mangle mounted on an optical microscope.

together with the maximum flux densities available when a/d $=1 / 4$. A zero field configuration at the centre is achieved by alternate rotation of the four rods through $\pm \phi=\pi / 2$ as shown in Fig 3(b). Any intermediate field can be achieved readily since $B(\phi)=B_{\max } \cos \phi$.

A magic mangle for an optical microscope has been designed and constructed on these principles. The magnets, of dimensions $\emptyset 25 \times 80 \mathrm{~mm}$, are fixed in copper cylinders, and each cylinder is mounted on ball bearings in the end plates. The axes lie on a square of side $54 \mathrm{~mm}$. The rotations of the four magnets are coupled by gears, and the angular position $\phi$ is set reproducibly within $0.5^{\circ}$ by means of a ten-turn potentiometer knob. Figure 4 illustrates the device mounted on a microscope. The variable field can be arranged to be either perpendicular or parallel to the microscope slide by mounting the mangle in the appropriate orientation.

The magnetic field was calculated from the vector potential $A(r)$ given by the Biot-Savart law, applied to the surface current densities equivalent to the permanent magnets. Figure 5 shows the calculated field profiles at maximum and zero field. In order to optimize the field in accordance with the finite length of the rods, it was advantageous to incline the rods by $\pm 11^{\circ}$, as shown in Figure 3(d).

When isotropic ferrite is used as the magnetic material, the maximum field achieved is $36.6 \mathrm{mT}$, with a homogeneity better than $1 \%$ within the central volume of diameter $7 \mathrm{~mm}$. The field at the zero setting is not so uniform as at the maximum. Homogeneity within $1 \%$ of maximum field is found in a volume of diameter $3.5 \mathrm{~mm}$ and a value of less than $0.1 \mathrm{mT}(1 \mathrm{G})$ is found within a volume of diameter $2 \mathrm{~mm}$. 

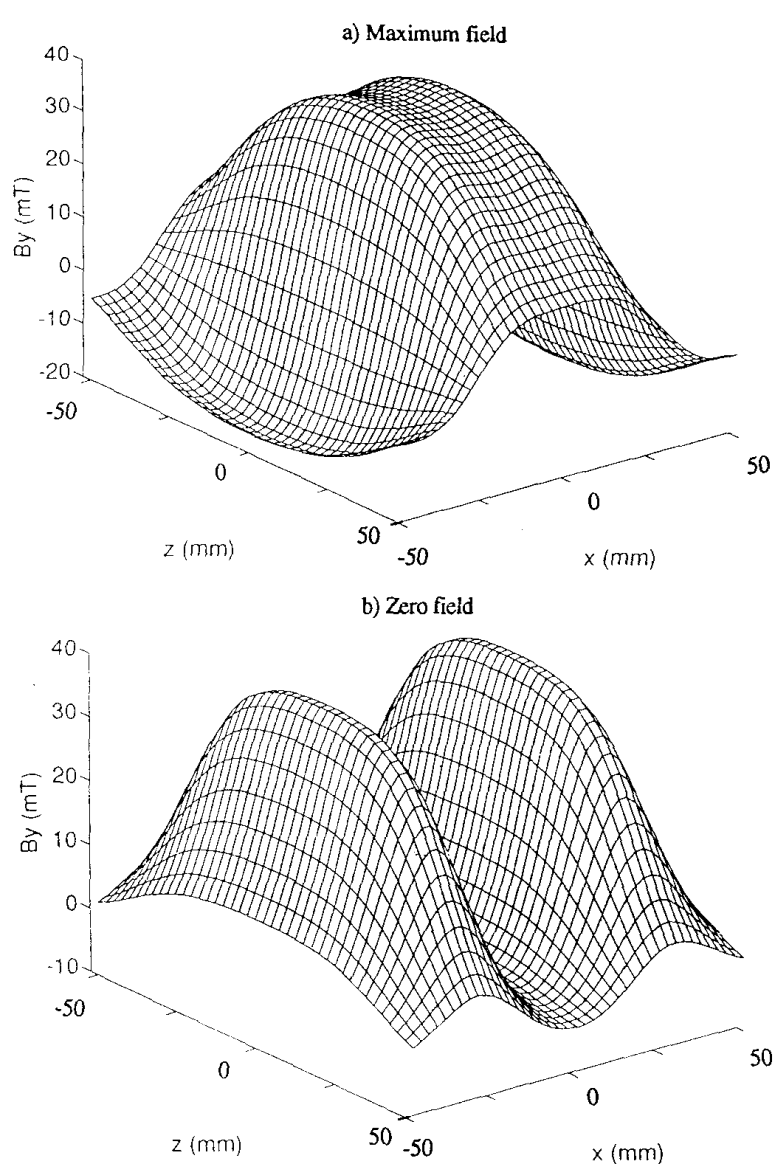

Fig 5: Calculated magnetic field profiles (a) at maximum field and (b) at zero field.

Comparison of calculated and experimentally-measured field profiles in Figure 6 shows excellent agreement between them.

The maximum field produced by the magic mangle is expected to scale with the remanence of the material used for the magnets (see Table 1). The fields experienced by each of the rods are negligible compared to its anisotropy field and much less than its coercivity. The maximum practicable field with the present geometry is therefore about $0.25 \mathrm{~T}(2.5 \mathrm{kG})$ if thehighest-remanence grade of Nd-Fe-B.is used. The field can be increased, if necessary, by increase of the magnet diameter and length, or by reduction of the gap. Designs with more than four rods offer improved field homogeneity [5].

An advantageous feature of the magic mangle is that it can be used to produce a low frequency alternating field at a few Hertz by attachment of a suitable motor to the drive shaft. This allows phase-sensitive lock-in detection of weak fielddependent signals associated, for example, with magneto-optic effects.

In conclusion, the magic mangle is a compact, simple and versatile solution to problems in optical microscopy requiring a uniform but variable magnetic field or magnetic field gradient at the specimen. Many other applications demanding a magnetic field with unobstructed access can be envisaged $[5,6]$

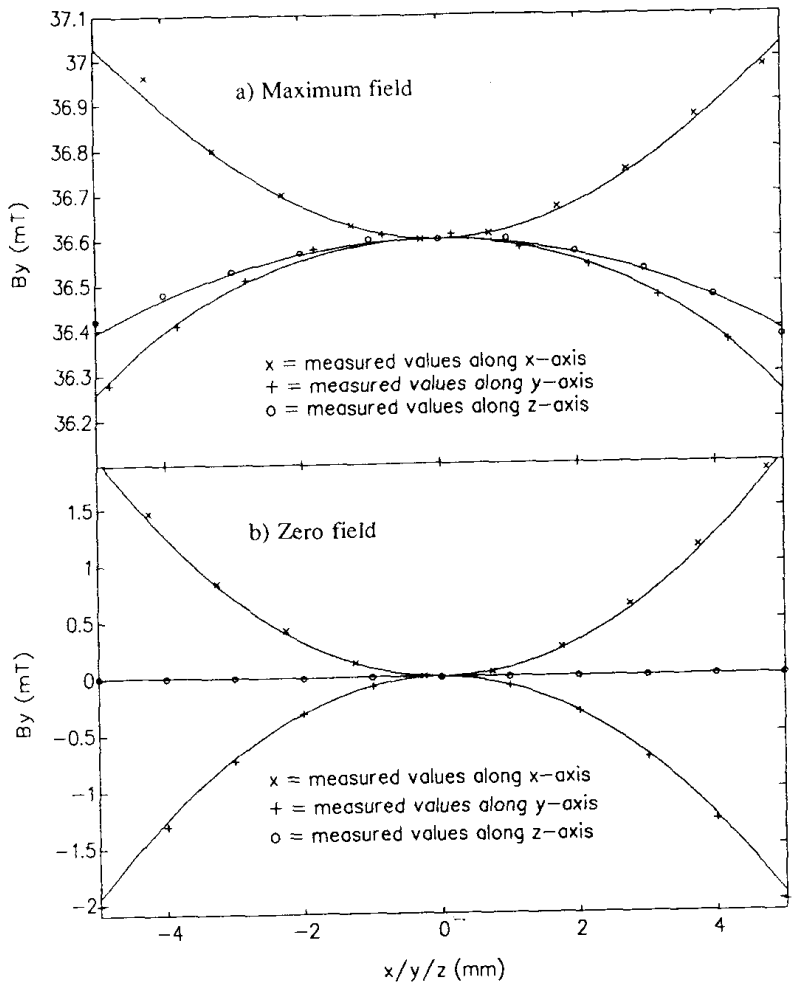

Fig 6: Comparison of the calculated (solid line) and measured (symbols) field profiles along a line passing through the midpoint of the mangle (a) at maximum field and (b) at zero field.

\section{ACKNOWLEDGMENT}

We are grateful to David Grouse for machining the magic mangle. This work forms part of the "Concerted European Action on Magnets", and Dr Orphée Cugat has benefited from a CEAM-HCM postdoctoral fellowship, supported by the European Commission.

\section{REFERENCES}

[1] M. G. Abele, Structures of Permanent Magnets, Wiley New York, 1993.

[2] H. Leupold in Rare-earth Iron Permanent Magnets, J M D Coey (editor), Oxford 1994 to be published.

[3] H..Leupold, E..Potenziani and M..G..Abele, "Applications of yokeless flux confinement," $J$ Appl Phys vol.64, pp 5994-5996, 1988

[4] O. Cugat, R. Byrne and J .M. D. Coey, "A compact vibratingsample magnetometer" Rev Sci Instrum vol.65 in press.

[5] J. M. D. Coey and O. Cugat, Variable permanent magnet flux sources, in Proc 9th Int Workshop on Rare-Earth Magnets and their Applications, Birmingham 1994 (in press).

[6] Custom-built permanent magnet flux sources are available from $M$ aglietic Nolutieu», Innovation Centre, Trinity College, Dublin 2, Ireland. 